Rev. Elev. Méd. vét. Pays trop. 1967, 20, 2 (279-289)

\title{
Prospections malacologiques aux Antilles françaises Observations sur l'écologie et l'élevage au laboratoire de Lymnaea cubensis Pfeiffer
}

\author{
par Simon GRÉTILLAT
}

\begin{abstract}
RÉSUMÉ
Des prospections malacologiques faites dans les cours d'eau et mares de la Martinique, de la Guadeloupe et de ses dépendances (Marie-Galante et lles des Saintes) permirent la récolte d'un certain nombre de gastéropodes d'eau douce : Lymnoeo cubensis Pfeiffer, Biomphalario hovanensis Pfeiffer, Australorbis peregrinus (d'Orbigny), Australorbis glabratus (Say); Drepanotrema cultratum (d'Orbigny), Physa mormorata Guilding, Potamopyrgus coronatus Pfelffer, Neretina punctulata Lamark. Quelques-uns d'entre eux n'avaient encore jamals été signalés dans ces îles.

Sur une souche de $L$. Cubensis rapportée de la Marlinique et mise en élevage à Dakar, on été éludiées quelques particularıtés écologiques de ce mollusque. Il peut vivre en dehors du milieu aquatique pendant longtemps. Pour le maintenir en élevage, il est nécessaıre de l'ınstaller dans des aquariums à fond vaseux avec eau irès aérée. Une alimentation riche, constituée de feuilles de laitue crue, accélère sa croissance et fournit des exemplaires de taille au-dessus de la normale qui perdent la propriété d'entrer en diapause quand le milieu se dessèche. Les individus alimentés seulement avec les matières organiques en suspension dans l'eau, se développent très lentement, mais gardent la faculté de pouvoir entrer en dıapause.
\end{abstract}

Tout récemment ont été reconnues la présence en Martinique, de Fasciola hepatica et celle de son vecteur Lymnaea cubensis Pfeiffer, mollusque fréquent aux Grandes Antilles, aux U. S. A., au Venezuela, mais sıgnalé pour la première fois dans cette île (GRÉTILLAT, 1966).

Les prospections malacologiques effectuées sur le terrain au sujet de l'épidémiologie de la douve du foie et de sa répartition géographique ont abouti à la récolte d'un certain nombre de gastéropodes d'eau douce récoltés dans des mares, ruisseaux ef torrents de la Martinique, de la Guadeloupe et de ses dépendances (MarieGalante et lles des Saintes).
Depuis 1945, plusieurs enquêtes ont été faites aux Antilles françaises sur la répartıtion géographique, la nature ef la fréquence des gîtes à Planorbes pouvant héberger les formes larvaires de Schistosoma mansom Sambon, 1907 agent causal de la bilharziose intestinale humane qui règne à l'état endémique dans ces îles (DESCHIENS ef Coll., 1955) (COURMES et Coll., 1964). Seul Australorbis glabrotus, vecteur de ce, schistosome, est signalé dans ces travaux.

En ce qui concerne l'helmınthologie vétérinaire, plusieurs affectıons à trématodes existent aux Antilles françaises. Il a donc paru intéressant de relever en vue de travaux ultérieurs, 
la fréquence et la répartition des mollusques d'eau douce, vecteurs éventuels de ces helminthiases (Fasciolose, Paramphistomose, Gastrodiscose).

La liste de ces gastéropodes avec la description sommaire de leur habitus, le ou les lieux de leur capture, leur fréquence, la nature de leur biotope avec quelques observations sur leur écologie sont données $\mathrm{cl}$-dessous.

La prophylaxie de la distomatose en Martinique, affection jusqu'à présent très peu répandue dans l'île, demande la mise en cuvre d'un programme visant à la destruction du mollusque vecteur : Lymnaea cubensis. Une étude de son écologie et de son comportement au laboratoire termine ce travail préliminaire sur la faune malacologique dulcaquicole des Antilles françaises.

\section{RÉSULTATS DES PROSPECTIONS MALACOLOGIQUES}

Celles-ci sont loin d'être complètes, mais apportent cependant une légère contribution à la connaissance des mollusques d'eau douce des Antilles françajses.

Lymnaea cubensis Pfeiffer (Fig. 1).

C'est le vecteur de Fosciola hepatica L. 1858 à La Martinique (GRÉTILLAT, 1966).

$\mathrm{Ce}$ mollusque à coquille dextre mesure 6 à $7 \mathrm{~mm}$ de long sur 2,6 à $3 \mathrm{~mm}$ environ. Gris plus ou moins clair, certains exemplaires sont ponctués de jaune sale sur la première convexité à partir de l'ouverture. Une fine striation visible seulement à la loupe en lumière rasante épouse les contours de la coquille qui a 5 tours de spires. Le pied massif est coiffé à sa partie antérieure de deux petites cornes trapues, aplaties de forme sensiblement triangulaire.

Gites d la Martinique : a) Belle Fontaine, dans un ruisseau et dans les cancux d'irrigation d'un pâturage artificiel à Digitoria decumbens. Très nombreux surtout dans les endroits où l'eau est fortement oxygénée (10 à 15 exemplaıres par $\mathrm{m}^{2}$ ) (GRÉTILLAT, 1966).

b) Rivière de I'Anse Charpentıer (côte est entre Marigot et Sainte-Marie) (com. R. P. PIN$\mathrm{CHON}$ ). A quelques centaines de mètres du lıttoral. Ce mollusque doit être fréquent dans les ruissecux de cetfe région. En effet, il y a quelques années, des cas de distomatose ont été observés par le Service vétérinaire des Abattoirs de Fort-de-France sur des bovins provenant du Lorrain.
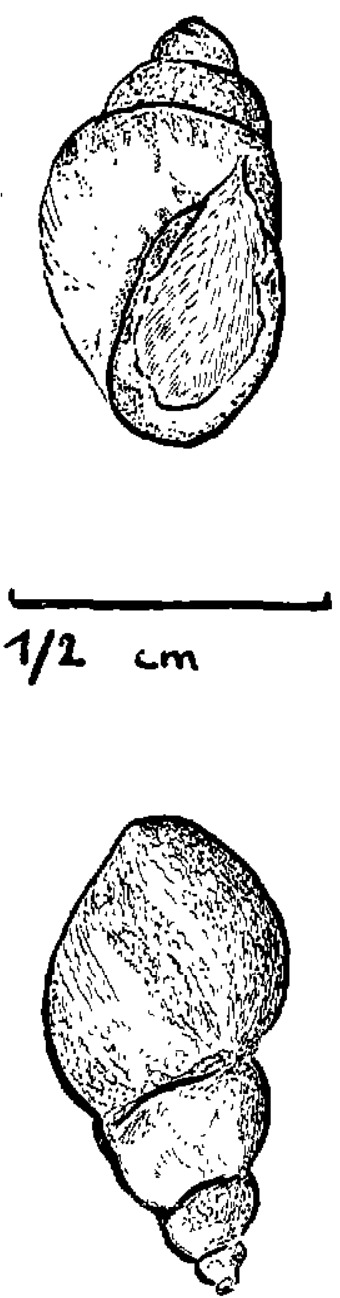

Fig. 1. - Lymnaea cubensis Pfeiffer.

c) Comme autre localisation probable des gîtes à L. cubensis, il y a lieu de citer les cours d'ecu des environs de Rivière Salée, pussque certains anımaux provenant de cette région sont atteints de fasciolose. Aucune prospection malacologique n'ayant encore été faite à ce sujet, il serait intéressant de rechercher ce gastéropode dans les nombreux canaux qui drannent les terrains de culture ef les pacages de basse altitude. 
Biomphalaria havanensis Pfeiffer (Fig. 2).

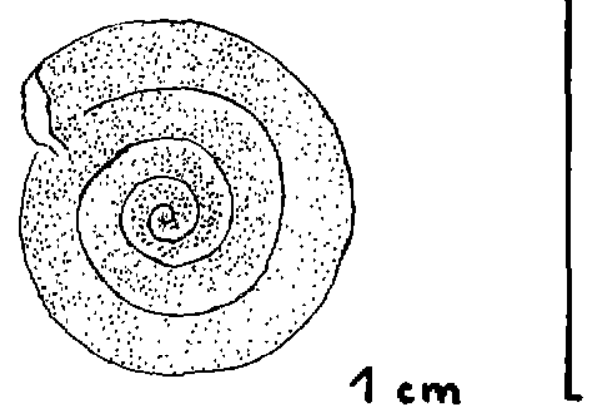

Fig. 2. - Biompholario hovonensis Pfeiffer.

Planorbe de taille moyenne 0,7 à $0,8 \mathrm{~cm}$ de diamètre à spires régulières, de couleur très foncée.

Semble surfout proliférer dans des eaux aérées ou renouvelées (equ de source), mais peut survivre dans des gîtes très vaseux, où sa multiplication semble alors arrêtée.

II fuit les milieux trop acides et marécageux.

II serait intéressant de rechercher dans quelle mesure, il peut être aux Antilles françaises un hôte intermédiaire de Schistosomo mansoni.

\section{Gîtes reconnus ef prospectés :}

10 Vallée du Fond Lailiet (Martinique). Dans conaux d'irrigation, en eau claire, sur fond sablo-argileux ou rocheux. $\mathrm{pH}=6,8$. Densité allant de 2 à 20 exemplaires par mètre carré.

20 Dans canaux d'irrigation des pacages de flancs de mantagne au Fond Laillet (Martinique). Très rares exemplaires.

3o Salines (Sud-Est de la Martınique) dans mares vaseuses, $\mathrm{pH}=7$. Eau très peu cérée. Végétation aquatique nulle. Rares exemplaires sans ponte.

40 Sainte-Anne (Martinique) dans une retenue d'eau artificielle; $\mathrm{pH}=6,8$. Végétation aquatique. Joncs et Cyperacées. Quelques exemplaires trouvés le long du barrage en maçonnerie et parmi les plantes.

50 Terrains de pacage des collines de la région du François (Martinique). Dans des mares vaseuses et surtout dans des abreuvoirs alimentés en eau par la canalisation urbaine. $\mathrm{pH}=6,8$.
Prolifération abondante dans ces collections d'eau créées par l'Homme (vieilles chaudières en fonte servant autrefois à la fabrication du rhum et utilisées actuellement comme abreuvoirs).

60 Mares dans terrains de pacages de la région du Robert (Martinique). Simples excavations où s'accumule l'eau de pluie. Le fond argileux imperméable en falt des points d'eau permanents dont certains sont alimentés partiellement par une petite résurgence.

Densité malacologique variable suivant le degré d'envasement et d'aération du milieu. 1 à 2 spécimens par mare.

Ces gîtes en état de repos en saison sèche (février-mars) doivent se repeupler dès le retour des pluies en avril-mai.

$7^{\circ}$ Canal d'irrigation d'un terrain de pacage à Baillif (Côte sous le Vent) (Guadeloupe) au flanc d'un contrefort montagneux dominant la mer. Eau claire, un peu courante, fond vaseux. 5 à 6 exemplaires par mètre carré de surface de gîte.

Australorbis peregrinus (d'Orbigny) (Fig. 3).

De taille plus grande que le précédent, sa coquille très foncée est plus épaisse, à ouverture large ef arrondie. Dimensions : 1 ì $1,2 \mathrm{~cm}$ de diamètre.

Au cours d'une prospection malacologique faite en collaboration avec I'Instituf Pasteur, la Direction de la Santé ef le Service des Eaux et Forêts de la Martinique dans la région de SaintPierre où règne une haute endémicité bilharzienne, ce gastéropode a été trouvé en grand nombre (20 à 50 mollusques par mètre carré de paroi) dans un bassin-piscine collectant les eaux d'un ruisseau (rivière Parnasse) à $7 \mathrm{~km}$ de Saint-Pierre. Ce bassın carré de $50 \mathrm{~m}$ de côté sur $2,50 \mathrm{~m}$ de profondeur, bâti en maçonnerie, est un endrat idéal pour la prolifération de A. peregrinus qui n'existe pas dans le canal d'amenée où l'eau est trop courante.

L'eau de ce bassin de $\mathrm{pH}=7$ est relativement claire et un peu aérée par renouvellement continuel en amont, sans végétation aquatique, mais les murs recouverts d'un épais tapis d'algues entretiennent une faune malacologique très dense.

Des tests de sortie de cercaires montrent que certains exemplaires hébergent les formes lar- 

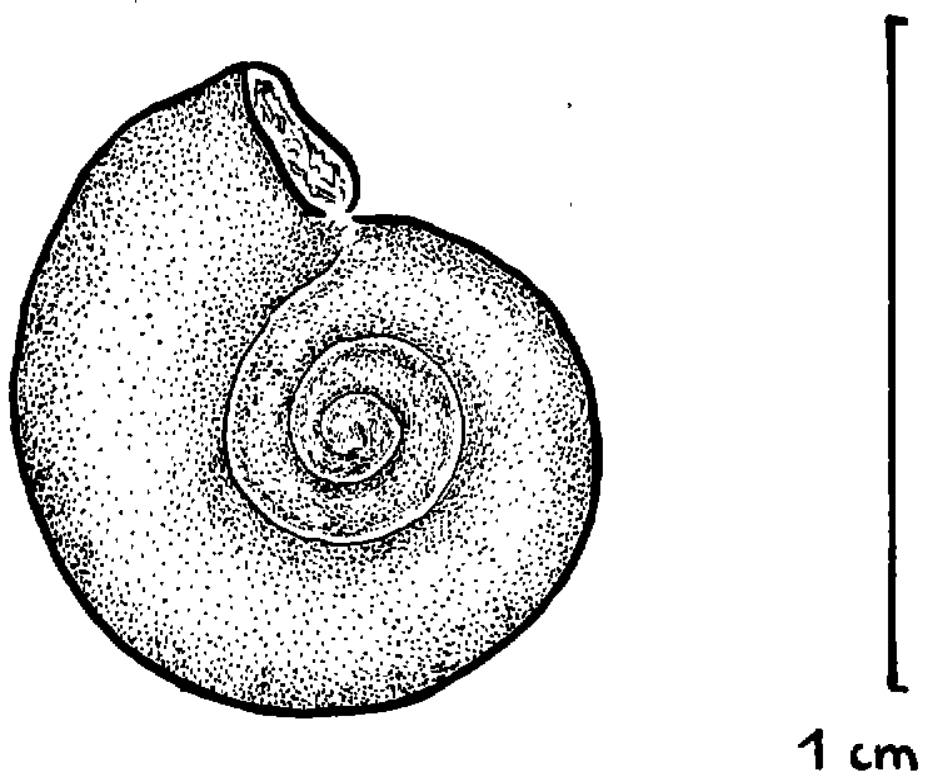

Fig. 3. - Australorbis peregrinus d'Orbigny.

vaires de Sch. mansoni. Ce réservoir d'eau est donc un foyer de schistosomiase intestinale humaine qu'il serait facile de détruire par épandage de molluscicides.

Cet exemple montre tout le danger que représente l'intervention de l'homme dans la construction ef l'aménagement des cours d'eau à des fins d'irrigation quand on ne prend aucune précaution pour éviter la prolifération des mollusques d'eau douce.

A la Martinique, en particulier, de nombreux gîtes à bilharziose ont été cinsi créés alors que dans les ruisseaux descendant de la montagne, les Austrolorbis sont très rares ou absents.

\section{Aastralorbis glabratus (Say) (Fig. 4).}

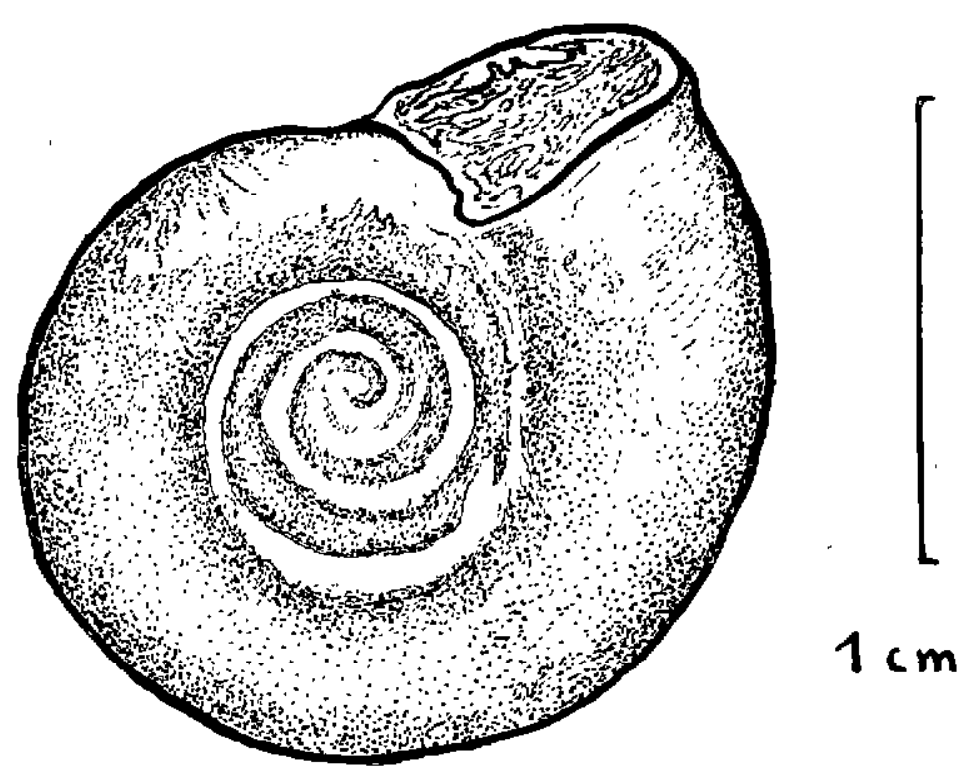

Fig. 4. - Australorbis glabrotus Say. 
Planorbe de très grande taille. Caquille noire, épaıse, à nombreux tours de spires. Dimensions : 1,5 à $2 \mathrm{~cm}$ de diamètre.

\section{Gîfes reconnus ef prospectés :}

10 Bassin d'une maison particulière au Robert (Martinique). Quelques exemplarres de très grande taille.

20 Fort-de-France, dans caniveaux de la ville basse.

30 Marie-Galante : mares de Pirogue, de Ducos, de Saint-Louis, de Grand-Bourg, de Vallon, de Ménard (Nord de l'lle).

Toutes ces mares qui sont pour la plupart des collections d'eau de pluie à fond argileux et dont le niveau baisse au cours de la saison sèche, sont des gîtes à $A$. glabrotus qui est au repos durant la période sèche. En effet, tous les exemplaires récoltés sont des adultes de très grande taille reposant sur la vase et en nombre relativement restreint.

40 Mares de la région est de la Grande-Terre (Guadeloupe), le long de la route du Moule à Saint-François, Mares de Zevalos, de Saint-Lovis. En vole d'assèchement avec quelques A. glabratus plus ou moins enfouis dans la vase des bords envahis souvent par des joncs.

50 Mare de Terre de Haut (Iles des Saintes) (Guadeloupe). De très nombreux $A$. glabratus adultes fixés aux racines et sous les feuilles de Pistia stratiotes $L$. qui recouvrent la surface de l'eau.

Des prospections malacologiques ayant été faltes à plusieurs reprises à la Guadeloupe au sujet de ce mollusque par DESCHIENS et Coll. en 1955, par MARILL en 1958, puis par COURMES et Coli. en 1964, les recherches sur le terrain n'ont pas été poussées plus loin.

\section{Drepanotrema cultrofum (d'Orbigny) (Fig. 5).}

Petit mollùsque ressemblant à une planorbe à coquille claire asymétrique. Il a une face plane sur laquelle il se tient au repos sur la vase ou sur des débris flottants (feuilles, branches tombées, etc...). Dimensıons : 0,6 à $0,9 \mathrm{~cm}$ de diamètre.

Ce gastéropode existe dans un étang permanent de Marie-Galante à $5 \mathrm{~km}$ au nord-est de Grand-Bourg (Mare Cosmobil).

C'est le premier Drepanotrema signalé des Antilles françaises.

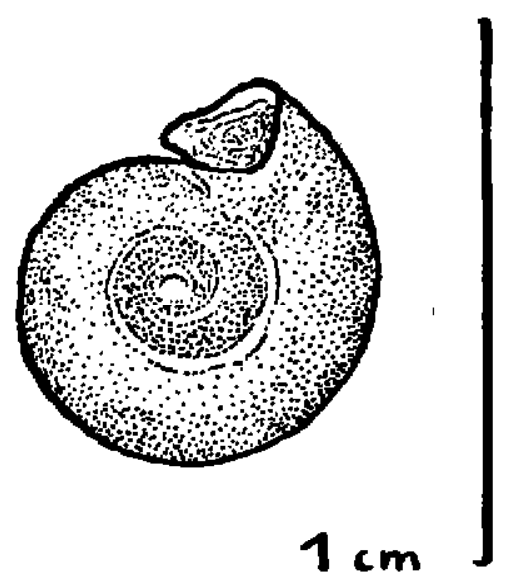

Fig. 5. - Drepanotrema cultratum d'Orbigny.

Physa marmorata Guilding (Fig. 6).

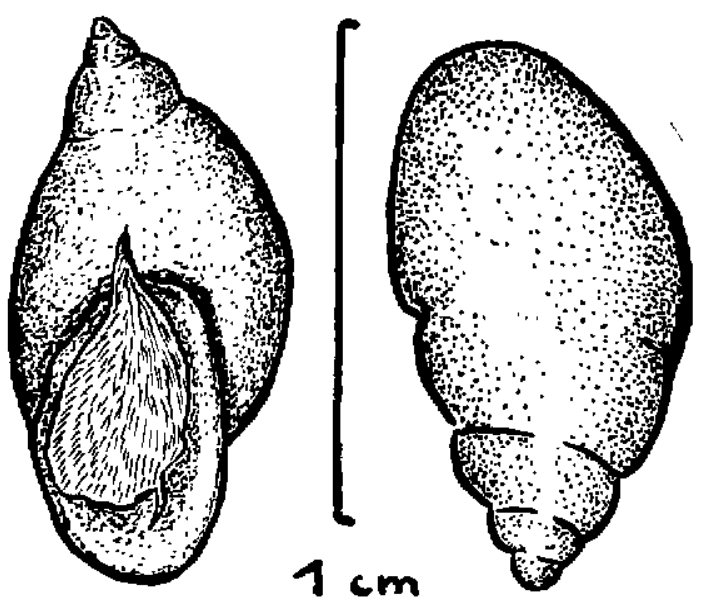

Fig. 6. -- Physa marmorato Guilding.

Mollusque à coquille allongée, claire, globuleuse en avant, à ouverture senestre, à columelle courte et pointue, très fréquent dans les points d'eau à la Martınıque et à la Guadeloupe où Il voisıne avec Bıompholarıo hovanensis, Australorbis glabratus et Lymnaea cubensis. Dımensions : 1 à $1,2 \mathrm{~cm}$ de long sur $0,5 \mathrm{~cm}$ d'épaisseur.

II prolıfère surtout dans les gîtes en eau claire légèrement oxygénée, à courant très faible avec un peu de végétation aquatique.

II serait intéressant de rechercher dans quelle mesure, il est susceptıble de transmettre certaines affections à trématodes des anımaux. 


\section{Gîtes reconnus et prospectés :}

10 Vallée du Fond Laillet (Belle-Fontaine, Martinique) canaux d'irrigation. 1 à 2 spécimens par mètre carré.

20 Pâturages de l'Elevage du Fond Laillet. Dans canaux d'irrigation à flanc de montagne 2 à 5 spécimens par mètre carré.

3o Mares de Salınes (Martinique). Très rares spécimens au repos dans points d'eau en voie d'assèchement.

40 Retenue d'eau d̀ Sainte-Anne (Martinique) fixée aux joncs des rives.

50 Mares de la région du Robert (Martinique). Très rares spécimens.

$6^{\circ}$ Le Baillif (Guadeloupe) (Côte sous le Vent) dans un canal d'ırrigation à flanc de montagne. Nombreux spécimens avec pontes en eau courante fixés sur les graminées.

Potamopyrgus coronatus Pfeiffer (Fig. 7).
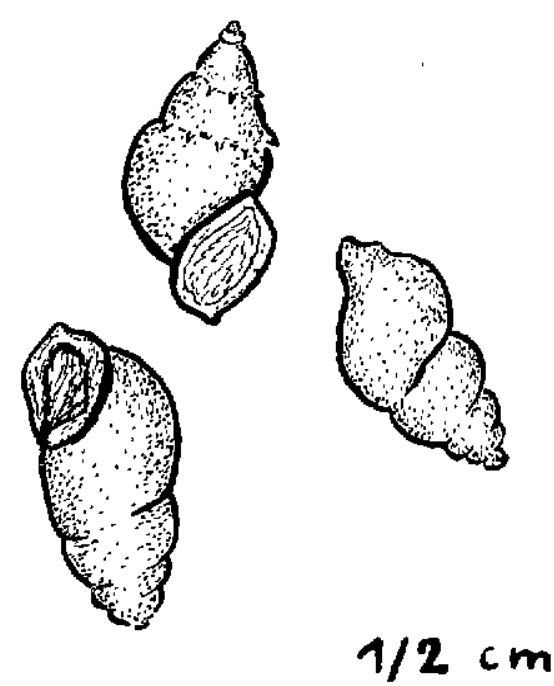

Fig. 7. - Potamopyrgus coronatus Pfeiffer.

Gastéropode d'eau douce operculé de très petite taille $(1,8$ à $2 \mathrm{~mm})$ à coquille brune, allongée, à ouverture dextre et à 5 tours de spires. Petit mollusque gastropade hydrobiidé ne devant pas être confondu avec Lymnaea cubensis qui est de taille double, à coquille plus clarre et dont les cornes sont courtes, plates et triangulaires alors qu'elles sont longues et filiformes chez $P$. coronatus. II existe des exemplaires à coquille lisse ef d'autres d̀ coquille épıneuse (cf. dessin).

Très nombreux exemplaires (20 à 30 par $\mathrm{m}^{2}$ ) avec pontes dans un petit ruisseau côtier de la «côte sous le vent» à $1 \mathrm{~km}$ au nord de Deshaies, « rivière de l'anse Mitan » le lang de la route de Sainte-Rose à Deshaies (Guadeloupe proprement dite). Eau courante claire, coulant sur fond vaseux avec lit du cours d'equ encombré de graminées aquatiques et de fragments de fevilles de canne d̀ sucre en voie de putréfaction servant de support et d'aliment aux mollusques.

Cet hydrobiidé existe à Porto-Rico ef aux U. S. Virgin Islands (FERGUSON et RICHARDS, 1963).

En 1965, DOBY, MANDAHL-BARTH, CHABAUD ef DEBLOCK on observé l'extrême proloficité de Potamopyrgus jenkisi (SMITH, 1889) dans certains torrents du sud de la Corse ou Bulinus truncatus était fréquent il y a encore quelques années (BUTTNER ef BOUCCART, 1957 ; GRÉTILLAT, 1963). D'introduction récente, $P$. jenkısı avait éliminé Bulinus truncatus de ces gîtes.

Il serait intéressant d'étudier dans quelle mesure Potomopyrgus coronatus serait susceptible de se comporter de la même manière avec Lymnaea cubensis, vecteur de Fasciola hepatica à la Martinique. En ce qui concerne la destruction d'Australorbis glabrotus par des moyens bialogiques, seul Marisa cornuarietis a donné des résultats satısfaisants à Porto-Rico (FERGUSON et Coll. 1958, RADKE ef Coll. 1951 ef autres).

Des recherches seraient d̀ entreprendre sur les possibilités de lutte biologique avec Potamopyrgus coronatus signalé pour la premıère fois à la Guadeloupe. Le dernier Comité d'Experts sur la bilharzıose de l'Organisation Mondiale de la Santé souligne l'intérêt et l'avantage d'un tel procédé dans le contrôle malacologique des viviers et exploitations aquicoles oùl'emplor des molluscicides chımiques est interdit pour des raisons pratiques.

Neretina punctulata Lamark.

Mollusque operculé, à coquille globuleuse, ornementé de points jaunâtres tranchant sur un fond sombre et fermé par un opercule épais.

Existe dans presque tous les torrents de la Martinique et de la Guadeloupe où on le trouve en plein courant fixé aux rochers. 


\section{PREMIÈRES OBSERVATIONS FAITES AU LABORATOIRE SUR L'ÉCOLOGIE DE Lymnaea Cubensis}

Si l'on s'en réfère à la bibliographıe, les quelques essais de mise en élevage de L. cubensis au Laboratoire ont été décevants, les auteurs ayant pu difficilement le maintenir en survie en utilisant certains artifices d'élevage tels que les aquaterrariums.

LEE en 1962, étudiant le cycle biologique d'Heterobilharzio americano Price, 1929, parasite du rancoon et d'autres mammifères du sud-est des U. S. A., constate que L. cubensis, hôte intermédiaire de ce trématode, s'adapte très difficilement aux conditions d'élévage en laboratoire. Pour réaliser le cycle d'H. americano, il se voit obligé de travailler sur des spécimens, infestés naturellement, récoltés dans des gîtes du Delta du Mississipi. LEE les maintient en survie en les nourrissant avec de la laitue fraîche. En aquarium, la plupart des spécimens s'enfouissent dans la vase humide où ils entrent en diapause.

VERGANI en 1955 au Venezuela avait déjà fait ceñe remarque. Après un repos de 235 jours, 45 p. 100 des mollusques sont capables de donner des pontes fertıles.

Avec beaucoup de précautions, il a été possıble de rapporter de la Marınıque, une souche de $L$. cubensis, maintenue en élevage depuis le 2 avril 1966.

Des 50 spécimens adultes récoltés dans un canal d'irrıgation de l'élevage du Fond Laillet (Belle Fontaine), le 9 mars 1966, il ne restait plus en arrivant à Dakar que 5 exemplaires dont 2 en très mauvais état, les autres étant morts au cours du transport ou au cours de leur séjour d̀ la Guadeloupe du 12 au 30 mars 1966.

Les différents milieux de survie ou d'élevage ont été successivement essayés :

0) Dans une equ de source non aérée artificiellement de $\mathrm{pH}=6,8$ et à $26^{\circ} \mathrm{C}$, L. cubensis fuit le milieu aquatique en quelques heures pour s'immobiliser sur une paroi sèche où Il entre en diapause. Remıs en eau dans les quelques heures qui suivent, Il reprend son activité pour fuir encore ef tenter de se remettre en diapause. Trois ou quatre fugues suivies d'une remise en aquarium aboutıssent à la mort du mollusque.

b) En eau de source non aérée avec fond rocheux, certains exemplaires s'immobilısent le pied fixé à la paroi rocheuse au niveau de la surface de l'eau, aux endroits où par capillarité le substrat est humide.

c) Dans le milieu précédent mais très fortement aéré par un oxygénateur à bulles, les mollusques se déplacent lentement et ont tendance à se rassembler aux endroits où l'aération est maximum.

Dans les trois milieux $a$ ), $b$ ), $c$ ), les mollusques délaissent la laıtue crue ou bouillıe qui leur est distribuée et aucune ponte n'est observée.

d) Des matières organiques en putréfaction ajoutées au milieu, telles que des fragments de feuilles de canne à sucre ayant séjourné dans l'eau pendant un certain temps, permettent de nourrir partiellement $L$. cubensis, qui ne fuit plus l'aquarium.

e) Sur fond vaseux ( $5 \mathrm{~cm}$ d'épaisseur) recouvert de $10 \mathrm{~cm}$ d'eau très fortement aérée, l'un des derniers survivants donne une ponte le $3 / \mathrm{V} /$ 66. De cet amas d'œufs (20 environ) en forme de virgule et de 1,5 à $2 \mathrm{~cm}$ de long, naissent le $12 / \mathrm{V} / 66,5$ Lymnaea cubensis qui sont la base de l'élevage.

Ces quelques exemplaires, ont, durant les deux premières semaines de leur vie, une croissance très lente. Se maintenant fixés aux parois de l'aquarium, à égale distance du fond de vase et de la surface, ils se nourrissent vraisemblablement des microorganismes en suspension dans l'eau. Becucoup moins « vagabonds » que leurs parents ramenés de la Martinique, ces jeunes L. cubensis commencent à manger de la laitue à partir de leur troisième semaine. Ils mesurent alors $2 \mathrm{~mm}$ de long. Deux semaines plus tard, ils ont atteint la taille des mollusques adultes qui leur ont donné nassance et commencent à pondre à l'âge de 34 jours. Le développement d'œuf à cuf a duré 43 jours, dont 9 pour l'éclosion des cufs, ce qui est extrêmement court.

Les pontes se succèdent nombreuses (26 pour 5 mollusques en 14 jours) et deviennent le déparì de cet élevage. La courbe de croissance (poids) donnée plus loın, a été établie à l'aıde d'exemplaires nés de ces pontes.

A partir du débuł de la période de ponte, la taille des mollusques s'accroît au point de dépasser en longueur 2 fois celle des $L$. cutensis récoltées sur le terraın, leurs poids étant environ 5 à 6 fois plus élevé. 
A 56 jours, ils mesurent 1,3 à $1,5 \mathrm{~cm}$ de long sur $0,60 \mathrm{à} 0,80 \mathrm{~cm}$ de large et pèsent en moyenne 0,28 à $0,35 \mathrm{~g}$.

Ces exemplarres élevés artificiellement ayant une taille anormale sans doute parce que soumis à un régime riche et régulier, il était intéressant de savoir sı leur faculté de résister à de mauvaises conditions du milieu, ne s'était pas amoindrie.

Pour ce faire, ils ont été successivement placés dans des aquariums où ont été progressivement supprimés, les éléments ayant permis leur croissance et leur multiplication (vase, laitue crue, aération artificielle):

10 Dans un aquarium en equ de source aérée, filtrée de $\mathrm{pH}=6,8$ à $24^{\circ} \mathrm{C}$, contenant de la laitue crue. Aucune tentative de fuite hors de l'aquarium n'est observée. Pendant une semaine, les mollusques contınuent à se nourrir en pondant régulièrement comme dans l'aquarium à fond vaseux.

20 Quand l'alimentation est supprimée mais I'aération du milieu maintenue, les $L$. cubensis ont un comportement normal et contınuent à pondre, mais certaines pontes sont stériles.

3o Au bout de 7 jours, l'aération est interrompue ; les mollusques s'immobilisent sur le fond et les parois de l'aquarium. Les pontes cessent, mais en une semaine, aucun exemplaire n'essaye de quitter le milieu aquatıque pour entrer en diapause.

40 Pour reconstituer au laboratore, les conditions d'assèchement d'un gîte, les mollusques sont installés dans un aquarium d̀ moitié rempli de vase humide avec en son centre un fragment de rocher dépassant de quelques centimètres la surface vaseuse. Dans ce nouveau milieu, les mollusques, après quelques déplacements de peu d'ımportance, s'ımmobilisent, après s'être à moitié enfouss dans la vase ou fixés sur la paroi rocheuse aux endroits où elle est un peu humide. Ils résıstent 3 jours ainsi, puis s'enfoncent tout à fait dans la vase, pour entrer en «diapause » ef résister à l'estivation, mais meurent au bout de six jours.

Par comparaison des lots de 20 Lymnaeo cubensis âgés de 3,4 et 5 semaines (alimentés avec laltue crue) (lots 1,2 et 3 ) et âgés de 5 semaines (sans laitue) (lot 4) ont été soumis à la même série de tests :
1) Lot de 3 semaines ... 80 p. 100 de mortalité 2) Lot de 4 semaines... 50 p. 100 de mortalité 3) Lot de 5 semaines ... 80 p. 100 de mortalité 4) Lot de 5 semaines... 30 p. 100 de mortalité sans alimentation artificielle.

Les individus très jeunes sont moins résistants à la dessiccation que les plus âgés. Une ałimentatıon artificıelle riche ef régulière amoindrit cette résistance.

\section{Croissance de $L$. cubensis dans les conditions d'élevage au Laboratoire.}

La courbe de croissance a été éfablie d'après le poids moyen de 25 exemplaires nés en aquarium sur fond vaseux dans une eau de $\mathrm{pH}=6,8$ fortement aérée, les mollusques étant nourris avec de la laitue crue.

Dans les quatre premières semaines, la croissance est régulıère. Les $L$. cubensis ne s'alimentent qu'avec des matıères organiques en suspension dans l'eau. La courbe prolongée suivant cette pente initiale donne un poids moyen de 30 d̀ $40 \mathrm{mg}$ à 8 semaines qui correspond à celui des spécimens adultes récoltés sur le terrain (courbe en pointillé).

A partir de la quatrième semaine, quand L. cubensis commence d̀ se nourrir avec de la laitue crue, la croissance s'accélère, le poids ef la taille dépassent la normale en deux semaines pour aboutır à des individus atteints de gigantisme (300 à $400 \mathrm{mg}$ d̀ 7 et 8 semaines).

\section{CONCLUSION}

Des observations faites sur le terrain et au cours de ces essars d'élevage en laboratoire, il ressort que :

10 L. Cubensis ne peut vivre que dans des eaux très oxygénées où l'eau est renouvelée constamment.

$2^{\circ}$ Un fand vaseux avec des matières organiques en suspension est indispensable à la survie, à l'alimentation et à la multiplication de L. cubensis.

30 Quand le milieu manque d'oxygène ou qu'וl se dessèche, $L$. cubensis le fuit pour entrer en diapause, en général en s'enfouissant dans le sol. 


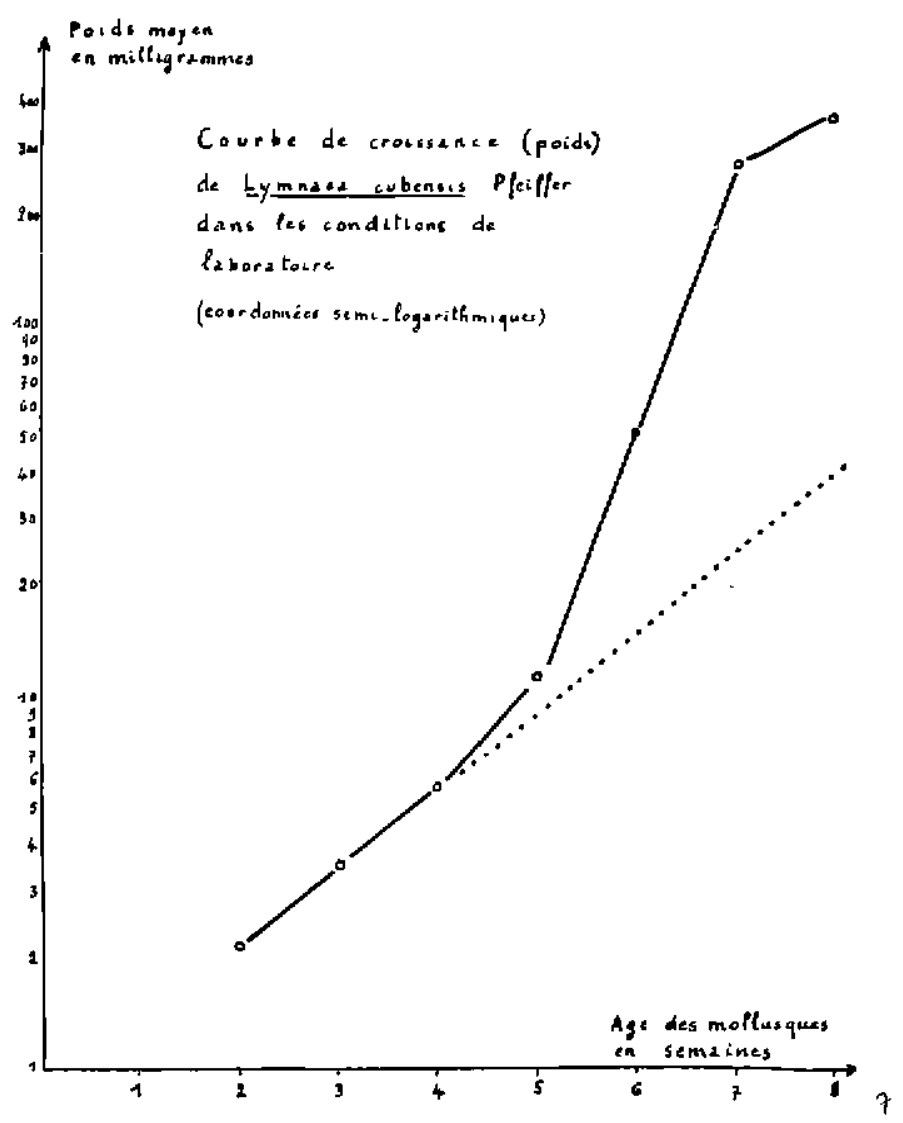

40 II est possible de maintenir en élevage L. cubensis à condition d'aménager un aquarium avec un fond vaseux épals de plusieurs centımètres ef d'aérer très fortement le milieu.

50 La croissance des $L$. cubensis en élevage est considérablement accélérée si on les alimente avec de la laitue crue. Ils délaissent la laitue bouillie. Des spécimens de la taille et du poids des adultes trouvés dans les gîtes naturels sont obtenus en 30 à 40 jours et commencent à pondre 35 à 40 jours après la naissance.

60 A partir du 35e jour, la taille et le poids de certains spécimens d'élevage dépassent la normale. Ces exemplaires résistent pendant plusieurs jours, à la suppression de toute alimentation, d'aératıon du milieu et leur activité dıminue progressivement.

70 Placés dans un terrarium de vase humide, ils survivent quelques jours au dessèchement puis meurent, sans avoir pu entrer en « diapause » pour estiver. Tout se passe comme si les conditions artificielles d'élevage, accélérant la croissance par une trop bonne alimentation, avaient fait disparaître la résistance de $L$. cubensis au dessèchement.

Lymnaea cubensis est un gastéropode, dont l'écologie un peu particulière pour une limnée, demande que certaines précautions soient prises lors des campagnes anti-mollusques. En état de diapause, il n'est pas détruit.

La souche martiniquaise semble assez ubiquiste en ce qui concerne son mode d'alimentation, et il est à craindre que ce mollusque ne colonise des cours d'eau ou ruisseaux où il n'existe pas encore. S'il envahissait des cressonnières, cela poserait un grave problème de santé humaine. Cependant, une alimentation riche et régulière fait apparaître des individus de taille et de poids dépassant largement la normale mais 
qui ont perdu la faculté de se mettre en état de diapause pour «estiver», Leur destruction par épandage de molluscicides est alors très facile.

\section{REMERCIEMENTS}

Le matériel malacologique a été déterminé par le Docteur MANDAHL-BARTH du Danish Bilharziasis Laboratory à Charlottenlund, Danemark.

Les prospections malacologiques et épidémiologiques ont été possıbles grâce à la collaboration du Docteur L. SAINT-PRIX du Service Vétérinaire de la Martinique, du R. P. PINCHON du Séminaire Collège de Fort-de-France, de
Messieurs DUVERGER et CLÉMENT de I'Institut de Recherches Agronomiques Tropicales à Fort-de-France, des Docteurs MILLE, LE GONIDEC et LABOURDETTE de l'Institut Pasteur de la Martinıque, de Messieurs BULIT et DIGAT du Centre de Recherches Agronomiques des Antilles, des Docteurs M.SAINT-PRIX, BAGÉ et BOSSARDET des Services Vétérinaires de la Guadeloupe et de Monsieur LIATARD du Service Vétérinaire de Marie-Galante.

\section{Institut d'Élevage et de Médecine Vétérnnaire des Pays tropicaux.}

Labaratoire Nahonal de l'Elevage ef de Recherches Vétérinaires de Dakar-Hann (Sénégal)

\section{SUMMARY}

Malacological survey in French Antilles.

Observations on ecology and laboratory rearing of Lymnaeo cubensis Pfeiffer Some malacological surveys have been made in the rivers and pools of Mortinique, Guadeloupe and Dependances (Marie-Galante and « lles des Saintes »), and some fresh water gasteropodes have been found: Lymnaea cubensis Pfeiffer, Bromphalaria havanensis Pfeiffer, Austratorbis peregrinus (d'Orbigny), Australorbis glabratus (Say): Drepanotrema cultratum (d'Orbigny), Physo marmorata Gulding, Potamopyrgus coronatus Pfeiffer, Neretina punctulato Lamark. Some of these had previously never been reported in those Islands.

Some of the ecological particularitıes of $L$. cubensis have been studied on a strain of this snail coming from Martinique and malntained for rearing purpose at Dakar. The snail was able to live a long tıme outside the water. To maintain it in rearing, it was necessary to keep it in aquaria with muddy boitom and well aerated water. The growth was accelerated by a rich food made of row lettuce leaves and the snails obtained by this method were bigger in size than the normal ones but they were not able to get into diapause when the environmental conditions became dry. The snails which had been fed only with organic substances in suspension in the water, grew very slowly but kept their ability to get into diapause.

\section{RESUMEN}

Investigaciones malacologicas en las Antillas francesas. Observaciones sobre la ecologia y la cria de Lymnaea cubensis Pfeiffer en el laboratorio

Se hicieron algunas investigaciones malacologicas en los ríos y charcas de la Martinica, de la Guadalupe y de sus dependencias (Maria-Galante y las Islas de las Santas) Se recogıó un cierta número de gasieropodos de agua : Lymnoea cubensis Pfeiffer, Blomphalaria hovanensis Pfeiffer, Australorbis peregrinus (d'Orbigny) Austrolorbis glabratus (Say). Drepanotremo cultratum (d'Orbigny) Physa marmorato Guilding, Potamopyrgus coronatus Pfeiffer, Neretina punctulata Lamark. Nunca se habian notado todavia algunos de ellos en dichas islas.

Se estudiaron algunas particularidades ecologicas de $L$. cubensis en una cepa de este molusco de Martinico criado en Dakar. Puede vivir fuera del medio acuático durante mucho tiempo. Para mantenerle en cria, se necesita 
ponerle en acuarios con fondo fangoso y agua muy aireada. Su crecimiento aumenta con una alımentación rica, constituida por hojas de lechuga cruda. Este método de cria da espécimenes de tamaño superıor al normal, perdiendo la propiedad de diapausia cuando el ambiente se deseca. El crecimiento de los moluscos solo alimentados con las materias orgánicas en suspensión en el agua es muy lento, pero se conserva la facultad de diapausia.

\section{BIBLIOGRAPHIE}

BUTTNER (A.) \& BOURCART (N.). - Etude des facteurs épidémiologiques qui président à la création d'un foyer de bilharziose humaine. Observations faifes au Brésil ef en Corse. Bull. Soc. Path. exot., 1957, 50 (3) : 473-480.

COURMES (E.), AUDEBAUD (G.) \& FAURAN $(P$.$) . - Distribution géographique locale$ d'Australorbis glabratus. Le problème épidémiologique qu'elle soulève à l'égard de la bilharziose intestinale dans les deux principales îles du département de la Guadeloupe. Bull. Soc. Path. exot., 1964, 67, 5 : 1071-1083.

DESCHIENS (R.), LAMY (L.) \& MAUZE (J.). Répartition géographique ef fréquence de la bilharziose intestinale en Guadeloupe. Bull. Soc. Path. exot., 1955, 46 : 810-819.

DOBY (Jean-Marie), MANDAHL-BARTH (Georg), CHABAUD (Alain), DEBLOCK (Stéphane). -- Elimination de Bulinus truncotus rivularis (Philippi) de collections d'eau connues pour l'héberger par Potamopyrgus jenkinsi (Smith, 1889) (Hydrobiidés), ef l'utilisation éventuelle de ce mollusque pour le contrôle biologique des bilharzioses. C. $R$. Acad. Sc., 1965, Paris 261, 4244-4246 (Séance du 15 novembre 1965).

FERGUSON (Frederick F.), GONZALEZ (José Olivier) \& PALMER (Juan R.). - Potential for biological control of Australorbis glabrafus the intermediate host of PuertoRican schistosomiasis. Amer. Journ. of Trop. Med \& Hyg., 1958, 7, 5 : 491-493.

FERGUSON (Frederick F.) \& RICHARDS (Charles S.). - Fresh-water mollusks of PuerioRico and the U.S. Virgin islands. Tronsactions of the American Microscopical Socrefy, 1963, 82 (4) : 391-395.

GRÉTILLAT (S.). - Epidémiologie de certaines affections à trématodes des animaux domes- tiques en Corse (bilharziose bovine ef distomatose bovine et ovine) (Observations effectuées au cours d'une mission réalisée en automne 1962). An. Parostt. hum. \& comp., 1963,38 (3) : 471-481.

GRÉTILLAT (5.). - Lymnoea cubensis Pfeiffer, mollusque hôte intermédiaire de Fasciola hepatica L., 1858 à la Martinique. C. R. Acod. Sci., 262 : 2736-39 (Séance du 27 Juin 1966).

GRÉTILLAT (S.). - Mission conjointe aux Antilles françaises, Martinique \& Guadeloupe. Enquête parasitologique (Helminthologie vétérinaire). Inst. Elev. Med. Vet. Pays Trop. \& inst. Nat. Rech. Agro., 1966, août 1966, 104 pages (Rapport non publié).

LEE (H. F.). - Life history of Heterobilharzia americana Price 1929 a schistosome of the rancoon and other mammals in south eastern United States. J. Porasit., 1962, 48 (5), : 728-739.

MARILL (F. G.). - Richesse en œufs des pontes de $P$. glabratus en Guadeloupe au cours de la saison sèche. Bull. Soc. Path. Exot.. 1958, 51, : 93 .

MOREL (P. C.). - Mission conjointe aux Antilles françaises, Guadeloupe \& Martinique. Enquête parasitologique (Entomologie \& Prołozoologie vétérinaire). Inst. Elev. Med. Vet. Pays Trop. \& Inst. Not. Rech. Agro., 1966, Rap. no 1/Parasit. 20/2/66, 112 pages (non publié).

RADKE (M. G.), RITCHIE (S.) \& FERGUSON (F. F.). - Demonstrated control of Australorbis glabrolus by Marisa cornuarietis under field conditions in Puerto-Rico. Amer. Journ. of Trop. Med. and Hyg., 1951, 10, 3, : 370-373. VERGANI $\left(F_{1}\right)$. - Datos biologicos experimentales sobre el caracol Limnoea (Galba) cubensis P. 1911. Boletin del Instifuto de Investigaciones Veterinarias, 1955, 7 (23) : 34-55. 